\title{
Identification of human limb stiffness in 5 DoF and estimation via EMG
}

\author{
D. Lakatos, D. Rüschen, J. Bayer, J. Vogel and P. van der Smagt
}

\begin{abstract}
To approach robustness and optimal performance, biological musculoskeletal systems can adapt their impedance while interacting with their environment. This property has motivated modern robotic designs including variable-impedance actuators and control methods, based on the capability to vary visco-elastic properties actively or passively. Even though variable-impedance actuation and impedance control in robotics is resolved to a great part, a general set of rules by which impedance is adjusted related to the task at hand is still lacking. This paper aims to fill this gap by providing a method to estimate the stiffness of the human arm in more than two degrees of freedom by perturbation. To overcome ill-conditionedness of the impedance and inertial matrices, we propose and validate methods to separately identify inertial and stiffness parameters. Finally, a model is proposed to estimate the joint stiffness from EMG-measurements of muscle activities.
\end{abstract}

\section{Motivation, Problem Statement, Related Work}

Dynamic interaction with the environment means handling impacts and unknown contact forces. Therefore compliant systems are active topics of research in the field of robotics. Surpassing traditional rigid robots, the control loops of modern robotic systems are extended with additional impedance parameters, viz. stiffness and damping.

Dominic Lakatos, Daniel Rüschen, Jörn Vogel and Patrick van der Smagt Institute of Robotics and Mechatronics, German Aerospace Center (DLR), D-82234 Oberpfaffenhofen, Germany e-mail: \{dominic.lakatos, daniel.rueschen, joern.vogel, smagt\}@ dlr.de

Justin Bayer

Chair for Robotics and Embedded Systems of the Department of Informatics of the Technische Universität München, D-80333 München e-mail: bayer . justin@googlemail .com. 
Even though the implementation of impedance control in robotics is resolved to a large part, one important issue still needs to be addressed: how are the impedance parameters set to optimally perform a task? Traditionally, robotic tasks are only defined in target end-effector positions or, in some cases, end-effector trajectories; but the impedance around these positions or trajectories remains a matter of common sense, at best. For instance, when performing a peg-in-hole task, high stiffness in the perpendicular and low stiffness in the lateral directions, so as to allow for imprecise positioning while solving the task, appears to be useful. But how do we find general rules-of-thumb for setting these extra parameters?

Beside heuristic methods tuning the impedance parameters, mimicking the behavior of the human arm is an auspicious field of research, and leads to what we call biologically-inspired robotics. By measuring and subsequently analyzing human arm impedance parameters, we can attempt to extract general rules and project these to the robotic domain.

The human arm's capability to alter its impedance has motivated multiple developments of robotic manipulators and control methods. It provides advantageous during manipulation such as robustness against external disturbances and task adaptability. However, how the impedance of the arm is set depends on the manipulation situation; a general procedure is lacking.

The only direct method to measure stiffness in a functioning feedback system is to apply external force perturbations to the limb and to measure the resulting displacements; such measurements have only been satisfactorily realized in planar (2D) movements $[8,12,4,5,10,2,11]$. To date, no fully satisfactory methods exist to investigate the time-varying impedance during movements. Early efforts were subject to error because they assume that subjects perform the same movement on repeated trials and they ignore the non-linear inertial properties of the musculoskeletal system.

We provide a method to identify human arm impedance in more than 2 degrees of freedom. We do this by initially identifying the kinematic and inertial parameters of the arm through movement. Subsequently we identify stiffness parameters of the human arm in 5 degrees of freedom (shoulder, elbow, and lower arm rotation), while taking the numerical stability of the data into account. The data are related to a representation of the stiffness by electromyography (EMG) signals which, in combination with the kinematics, gives us a 3D Cartesian identification of the impedance parameters of the human arm.

\section{Technical Approach}

An adequate model describing the human limb dynamics can be separated in two power interconnected subsystems: the mass inverse dynamics of the skeleton (including the mass distribution of the muscles)

$$
\boldsymbol{\Gamma}(\boldsymbol{q}, \dot{\boldsymbol{q}}, \ddot{\boldsymbol{q}}, \boldsymbol{\xi})=\boldsymbol{\tau}+\boldsymbol{\tau}_{\mathrm{ext}},
$$


where $\boldsymbol{q} \in \mathbb{R}^{n}$ are joint positions, $\boldsymbol{\xi} \in \mathbb{R}^{\leq 10 n}$ are base inertial parameters and $\tau_{\text {ext }}$ are external torques and general impedance functions of the muscular system, acting as force elements on the joints:

$$
\boldsymbol{\tau}=-\boldsymbol{h}(\boldsymbol{q}, \dot{\boldsymbol{q}}, \boldsymbol{a}) .
$$

We assume $\boldsymbol{h}: \boldsymbol{q}, \dot{\boldsymbol{q}}, \boldsymbol{a} \rightarrow \boldsymbol{\tau}$ to be continuous, while the muscle activities $\boldsymbol{a}$ are motor commands, which are able to shift the equilibrium point of the impedance. Thus, linearization in the working point $\boldsymbol{x}_{d}:=(\boldsymbol{q}(t=0), \dot{\boldsymbol{q}}(t=0), \boldsymbol{a}(t=0))$ yields:

$$
\boldsymbol{h}^{\star}=\underbrace{\left.\boldsymbol{h}\right|_{\boldsymbol{x}_{d}}}_{\tau_{d}}+\underbrace{\left.\frac{\partial \boldsymbol{h}(\boldsymbol{q}, \dot{\boldsymbol{q}}, \boldsymbol{a})}{\partial \boldsymbol{q}}\right|_{\boldsymbol{x}_{d}}}_{\boldsymbol{K}_{q}} \tilde{\boldsymbol{q}}+\underbrace{\left.\frac{\partial \boldsymbol{h}(\boldsymbol{q}, \dot{\boldsymbol{q}}, \boldsymbol{a})}{\partial \dot{\boldsymbol{q}}}\right|_{\boldsymbol{x}_{d}}}_{\boldsymbol{D}_{q}} \dot{\boldsymbol{q}}+\left.\frac{\partial \boldsymbol{h}(\boldsymbol{q}, \dot{\boldsymbol{q}}, \boldsymbol{a})}{\partial \boldsymbol{a}}\right|_{\boldsymbol{x}_{d}} \tilde{\boldsymbol{a}}+\ldots
$$

Additionally, we assume that activations $\boldsymbol{a}=$ const. (this can be fulfilled by certain experimental conditions); consequently the joint torques acting due to muscles can be approximated by:

$$
\boldsymbol{\tau}=-\boldsymbol{\tau}_{d}-\boldsymbol{K}_{q} \tilde{\boldsymbol{q}}-\boldsymbol{D}_{q} \dot{\tilde{\boldsymbol{q}}},
$$

where $\tilde{\boldsymbol{q}}=\boldsymbol{q}-\boldsymbol{q}_{d}$ is the tracking error, $\boldsymbol{\tau}_{d}$ are equilibrium torques and $\boldsymbol{K}_{q}, \boldsymbol{D}_{q}$ are joint stiffness and damping matrices, respectively.

Identifying the complete parameter set (i.e., $\boldsymbol{\xi}, \boldsymbol{K}_{q}$ and $\boldsymbol{D}_{q}$ ) from measurements of $\tau_{\text {ext }}$ would lead to an ill-conditioned least-squares problem [7], thus we estimate $\xi$ separately by projecting the inertial forces to the subject's mounting base where they can be measured with a force / torque sensor, i.e.,

$$
\chi_{0}(\boldsymbol{q}, \dot{\boldsymbol{q}}, \ddot{\boldsymbol{q}}, \boldsymbol{\xi})=\chi_{\text {sensor }} .
$$

Once the inertial parameters $\boldsymbol{\xi}$ are known, the not directly measurable joint torques $\tau$ can be estimated via the inverse dynamic model and the identification model reduces to

$$
\boldsymbol{K}_{q} \tilde{\boldsymbol{q}}+\boldsymbol{D}_{q} \dot{\tilde{\boldsymbol{q}}}=\boldsymbol{\tau}_{\mathrm{ext}}-\boldsymbol{\Gamma}(\boldsymbol{q}, \dot{\boldsymbol{q}}, \ddot{\boldsymbol{q}}, \boldsymbol{\xi})-\boldsymbol{\tau}_{d},
$$

where only the left hand side is unknown. This separation allows severed identification of parameters for each subsystem - to overcome the problem of badly scaled least-squares estimations [7].

The complete 5-DoF identification procedure requires the following steps:

1. identification of center of rotation for the 3-DoF shoulder joint and 2-DoF elbow joint, respectively;

2. solving inverse kinematics, which gives an approximation of the Jacobian matrix;

3. estimating the inertial parameters via kinematics data and base force / torque sensing;

4. separated identification of the impedance parameters while EMG signals of the active muscles are recorded;

5. train a model in order to predict stiffness from EMG data which finally gives the possibility to estimate stiffness without mechanical measurements. 


\subsection{Kinematic identification}

To identify the positions of the human limb joints, a method proposed by [1] is conducted. We assume that at least two axes of rotation intersect. The absolute position $\boldsymbol{r}$ of the point of intersection can be represented via markers placed at the joint adjacent body segments, i.e.

$$
\begin{aligned}
& \boldsymbol{r}_{1}=\boldsymbol{p}_{1}+\boldsymbol{R}_{1}^{T} \boldsymbol{d}_{1}, \\
& \boldsymbol{r}_{2}=\boldsymbol{p}_{2}+\boldsymbol{R}_{2}^{T} \boldsymbol{d}_{2},
\end{aligned}
$$

where $\boldsymbol{p}_{i}$ and $\boldsymbol{R}_{i}$ denotes the absolute position and orientation of a marker and $\boldsymbol{d}_{i}$ is the distance of the intersection point w.r.t. the $i$ th marker. Thus minimizing the integral error

$$
S=\frac{1}{T} \int_{0}^{T}\left(\boldsymbol{r}_{1}-\boldsymbol{r}_{2}\right)^{T}\left(\boldsymbol{r}_{1}-\boldsymbol{r}_{2}\right) \mathrm{d} t,
$$

determines the unknown $\boldsymbol{d}_{1}$ and $\boldsymbol{d}_{2}$.

\subsection{Inverse Kinematics}

The kinematics of the human limb consists of uncertainties, e.g., non-ideal joints and varying segment lengths. In order to minimize these errors we propose a numerical solution of the inverse kinematics, i.e.

$$
\arg \min \left\|\boldsymbol{T}(\boldsymbol{q}) \boldsymbol{T}_{d}^{-1}-\boldsymbol{I}\right\|_{F},
$$

where $\boldsymbol{T}(\boldsymbol{q})$ and $\boldsymbol{T}_{d}$ are the parameterized and desired homogeneous transformation matrix to the wrist, respectively and $\|.\|_{F}$ denotes the Frobenius matrix norm. This optimization problem is continuous and unconstrained and can be solved with, e.g., a quasi-Newton method.

\subsection{Inertial parameter model}

For the identification of the inertial parameters, a model can be considered where the dynamical forces / torques are projected to a coordinate system at the subject's mounting base, i.e., under the seat (a similar approach was proposed by [13]). The equations of this model can be obtained analytically by means of the iterative Newton-Euler formalism:

$$
\begin{gathered}
\chi_{i}=\left[\begin{array}{c}
\boldsymbol{f}_{i} \\
\boldsymbol{n}_{i}
\end{array}\right]=\left[\begin{array}{c}
\boldsymbol{F}_{i}(\boldsymbol{q}, \dot{\boldsymbol{q}}, \ddot{\boldsymbol{q}}, \boldsymbol{\xi})+\boldsymbol{R}_{i, i+1}(\boldsymbol{q}) \boldsymbol{f}_{i+1} \\
\boldsymbol{N}_{i}(\boldsymbol{q}, \dot{\boldsymbol{q}}, \ddot{\boldsymbol{q}}, \boldsymbol{\xi})+\boldsymbol{R}_{i, i+1}(\boldsymbol{q}) \boldsymbol{n}_{i+1}+\widetilde{\boldsymbol{p}}_{i, i+1}(\boldsymbol{q})\left(\boldsymbol{R}_{i, i+1}(\boldsymbol{q}) \boldsymbol{f}_{i+1}\right)
\end{array}\right], \\
\text { for } \quad i=n_{\text {body }}, n_{\text {body }}-1, \ldots, 0,
\end{gathered}
$$


where $\chi_{i}$ is the wrench acting on the $i$ th body. $\boldsymbol{R}_{i, i+1}$ and $\boldsymbol{p}_{i, i+1}$ are the relative rotation and distance between body $i$ and $i+1$, respectively. Furthermore, $\boldsymbol{f}_{i+1}$ and $\boldsymbol{n}_{i+1}$ are Cartesian forces and torques propagated from the $(i+1)$ st body. Finally, $\boldsymbol{F}_{i}$ and $\boldsymbol{N}_{i}$ are the forces and torques due to the inertial dynamics. They can be computed by:

$$
\begin{aligned}
\boldsymbol{F}_{i} & =m_{i} \dot{\boldsymbol{v}}_{i}+\dot{\overline{\boldsymbol{\omega}}}_{i} \boldsymbol{S}_{i}+\widetilde{\boldsymbol{\omega}}_{i}\left(\widetilde{\boldsymbol{\omega}}_{i} \boldsymbol{S}_{i}\right), \\
\boldsymbol{N}_{i} & =\boldsymbol{\Theta}_{i} \dot{\boldsymbol{\omega}}_{i}+\widetilde{\boldsymbol{\omega}}_{i}\left(\boldsymbol{\Theta}_{i} \boldsymbol{\omega}_{i}\right)+\widetilde{\boldsymbol{S}}_{i} \dot{\boldsymbol{v}}_{i} .
\end{aligned}
$$

Herein $\widetilde{\boldsymbol{\omega}} \in \mathbb{R}^{3 \times 3}$ denotes the skew symmetric tensor composed of components $\omega \in \mathbb{R}^{3} . \boldsymbol{v}_{i}, \omega_{i}$ and $\dot{\boldsymbol{v}}_{i}, \dot{\omega}_{i}$ are absolute translational and angular velocities and accelerations of the $i$ th body. The inertial parameters $m_{i} \in \mathbb{R}, \boldsymbol{S}_{i} \in \mathbb{R}^{3}$, and $\boldsymbol{\Theta}_{i} \in \mathbb{R}^{3 \times 3}$ (i.e., mass moments zeroth, first, and second order) are linear in the base base projected model $\chi_{0}(\boldsymbol{q}, \dot{\boldsymbol{q}}, \ddot{\boldsymbol{q}}, \boldsymbol{\xi})=\chi_{\text {sensor }}$ and can be identified by common least-squares estimations.

\subsection{Impedance identification}

Due to the constraint of energy conservation the force field generated by mechanical stiffness must be integrable [6], i.e., stiffness matrices are symmetric and positive definite (SPD). In order to enhance the robustness of the identification procedure we also determine the stiffness separated from damping. Therefore we take the reduced model

$$
\boldsymbol{K}_{q} \tilde{\boldsymbol{q}}=\boldsymbol{\tau}_{\mathrm{ext}}-\boldsymbol{\Gamma}\left(\boldsymbol{q}, \boldsymbol{\xi}_{\mathrm{red}}\right)-\boldsymbol{\tau}_{d},
$$

into account. This model is valid for the stationary case $\ddot{\boldsymbol{q}}=\dot{\boldsymbol{q}} \approx 0$. In that case the stiffness balance the (non-linear) gravity and external torques.

The identification model is linear in $\boldsymbol{K}_{q}$ and consists of the form

$$
\boldsymbol{A} \boldsymbol{X}=\boldsymbol{B},
$$

where $\boldsymbol{A}=\tilde{\boldsymbol{q}}^{T}, \boldsymbol{X}=\boldsymbol{K}_{q}^{T}$ and $\boldsymbol{B}=\left(\boldsymbol{\tau}_{\mathrm{ext}}-\boldsymbol{\Gamma}\left(\boldsymbol{q}, \boldsymbol{\xi}_{\text {red }}\right)-\boldsymbol{\tau}_{d}\right)^{T}$. To ensure the SPD constraint the area criterion proposed by [3]:

$$
f(\boldsymbol{Y})=\left\|\boldsymbol{A} \boldsymbol{Y}-\boldsymbol{B} \boldsymbol{Y}^{-T}\right\|,
$$

where $\boldsymbol{X}=\boldsymbol{Y} \boldsymbol{Y}^{T}$, will be minimized. When $\boldsymbol{P}=\boldsymbol{A}^{T} \boldsymbol{A}$ and $\boldsymbol{Q}=\boldsymbol{B}^{T} \boldsymbol{B}$ the unique solution is given by

$$
\hat{\boldsymbol{K}}_{q}=\hat{\boldsymbol{X}}=\boldsymbol{U}_{P} \Sigma_{P}^{-1} \boldsymbol{U}_{\tilde{Q}} \Sigma_{\tilde{Q}} \boldsymbol{U}_{\tilde{Q}}^{T} \Sigma_{P}^{-1} \boldsymbol{U}_{P}
$$

where

$$
\begin{aligned}
\boldsymbol{P} & =\boldsymbol{U}_{P} \Sigma_{P}^{2} \boldsymbol{U}_{P}^{T}, \\
\tilde{\boldsymbol{Q}}=\Sigma_{P} \boldsymbol{U}_{P}^{T} \boldsymbol{Q} \boldsymbol{U}_{P} \Sigma_{P} & =\boldsymbol{U}_{\tilde{Q}} \Sigma_{\tilde{Q}}^{2} \boldsymbol{U}_{\tilde{Q}}^{T},
\end{aligned}
$$


are the Schur decompositions of $\boldsymbol{P}$ and $\tilde{\boldsymbol{Q}}$, respectively.

\subsection{Stiffness determination from EMG}

To predict a stiffness matrix $\boldsymbol{Z}_{i}$ from EMG data $\boldsymbol{x}_{i}$, a nonlinear two-layer model was used. As $\boldsymbol{Z}_{i}$ is symmetric and positive definite, we note that it can be decomposed into $\boldsymbol{Z}_{i}=\boldsymbol{L}_{i} \boldsymbol{L}_{i}^{T}$ via the Cholesky decomposition.

Constraining the output of our model to be positive definite and symmetric can thus be done by not modeling $\boldsymbol{Z}_{i}$, but $\boldsymbol{L}_{i}$ instead.

In more detail, given $N$ time windows $\left\{\boldsymbol{x}_{i}\right\} \subset \mathbb{R}^{n \times m}$ where $n$ is the length of the time windows and $m$ is the number of EMG electrodes, we predict the components of the Cholesky decomposition via

$$
\boldsymbol{l}_{i}=\frac{1}{N} \boldsymbol{W}_{1} \sigma\left(\boldsymbol{W}_{2} \phi\left(\boldsymbol{x}_{i}\right)+\boldsymbol{b}_{2}\right)+\boldsymbol{b}_{1},
$$

where $\phi$ is a function that extracts features from each time window and $\sigma$ is a nonlinear function applied component-wise. We then turn $\boldsymbol{l}_{i}$ into a lower-triangular matrix $\boldsymbol{L}_{i}$ by rearranging the components from vector into matrix form. The final prediction is subsequently formed by $\boldsymbol{Y}_{i}=\boldsymbol{L}_{i} \boldsymbol{L}_{i}^{T}$.

The parameters of the model $\boldsymbol{\theta}=\left\{\boldsymbol{W}_{1}, \boldsymbol{W}_{2}, \boldsymbol{b}_{2}, \boldsymbol{b}_{1}\right\}$ are either matrices $\boldsymbol{W}_{1}$ and $\boldsymbol{W}_{2}$ or vectors $\boldsymbol{b}_{1}$ and $\boldsymbol{b}_{2}$. To learn such model, we assume that the measurements of the stiffness matrices $\left\{\boldsymbol{Z}_{i}\right\}$ are subject to Gaussian noise and minimize the negative log likelihood:

$$
\log \lambda \cong \sum_{i}\left\|\boldsymbol{Y}_{i}-\boldsymbol{Z}_{i}\right\|^{2} .
$$

The resulting optimization problem is unconstrained and continuous. The gradients are efficiently computed via dynamic programming and the chain rule. Thus, standard off-the-shelf optimizers are used to find good solutions for $\boldsymbol{\theta}$.

\section{Experiments}

During the whole experiment subjects were seated on a special chair depicted in Figure 1(a) while the upper body was restrained by a seat belt. At the wrist a plastic cuff supported the connection to the robot's end-effector. JR3 force / torque sensors were placed at the interconnection (between robot and limb) and at the subject's mounting base (under the seat). The data of both force / torque sensors were sampled at $2 \mathrm{kHz}$. To estimate the kinematic configuration optical tracking markers were placed at the upper body, upper arm and forearm, respectively. We used data from Vicon T10 cameras to track the markers position and orientation, sampled at $500 \mathrm{~Hz}$. To map EMG to stiffness, we recorded EMG signals from eight sources on the arm 
(see Fig. 1(b)). We used Delsys Trigno wireless electrodes, sampled at $2 \mathrm{kHz}$. Experimental instructions and visual feedback were given to the subject via a display.

At the beginning of an experimental session, data from the optical tracking system was recorded to identify the subject's individual joint positions and compute the arm kinematics. Here the subject was instructed to move all joint axes of the limb randomly. After this, data (40 trials) for the inertial parameters were gathered, while different predefined kinematic limb configurations had to be reached in free movement. These initial recordings where followed by the final stiffness identification procedure in which subjects had to fulfill a force task. The subject's wrist was coupled to the light-weight robot's end-effector and desired and actual interaction forces / torques were displayed. After holding a certain force / torque level (4 levels in Cartesian $\mathrm{X}$ and $\mathrm{Z}$ direction each) for a random duration between 1.5 and 2.5 seconds, the robot perturbs the limb in one direction randomly chosen from the 10 possibilities (two for each joint DoF). All smooth (polynomial fifth order) displacements were planned in joint coordinates of the human limb with an amplitude of $\approx 0.08 \mathrm{rad}$ via the Jacobian matrix, i.e., $\Delta \boldsymbol{x}_{\text {robot }}=\boldsymbol{J}_{\mathrm{limb}}(\boldsymbol{q}) \Delta \boldsymbol{q}$. Typical disturbances are shown in Figure 2.

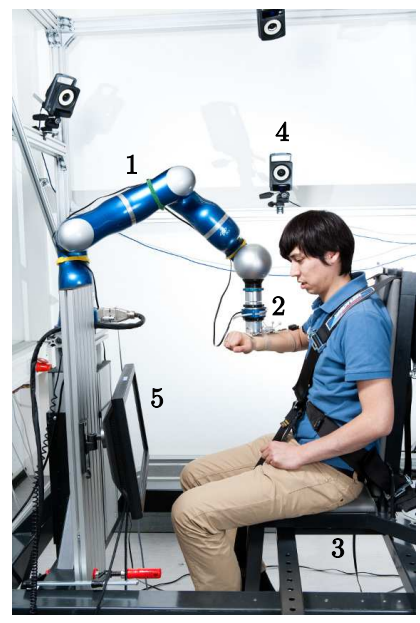

(a)

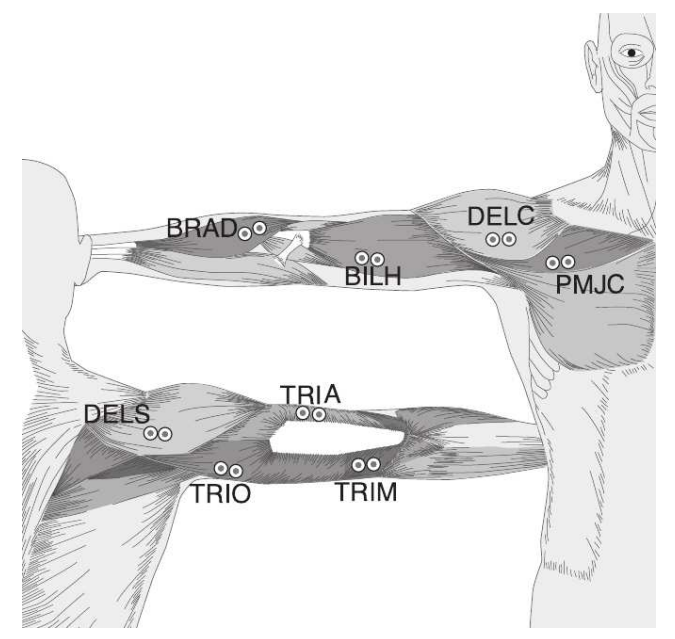

(b)

Fig. 1 a Experimental setup: (1) DLR light-weight robot applies disturbances to the human arm, (2) JR3 force / torque sensor measures interaction forces, (3) JR3 force / torque sensor measures subject's mounting base forces, (4) Vicon T10 optical tracking system, (5) Subject's visual feedback. b EMG electrode placement for estimating stiffness from EMG. A total number of 8 electrodes are placed. EMG signals of dominant muscles involved in shoulder and elbow joint movements are gathered: brachioradialis $(B R A D)$, biceps long $(B I L H)$, deltoid clavicular $(D E L C)$, pectoralis major clavicular $(P M J C)$, deltoid scapular $(D E L S)$, triceps long $(T R I O)$, triceps lateral (TRIA), and triceps medial (TRIM). 


\section{Results}

\subsection{Estimated stiffness and prediction via EMG}

After estimating the joint positions, the inverse kinematics of all trials (i.e., inertial and stiffness measurements) were computed. First identification results are obtained from stationary parts of the recorded data, i.e., where the velocity is lower than a certain threshold. Therefore the inertial identification model reduces to

$$
\chi_{0}\left(\boldsymbol{q}, \boldsymbol{\xi}_{\text {red }}\right)=\chi_{\text {sensor }}
$$

where $\xi_{\text {red }}$ contains the mass moments zeroth and first order. To estimate $\boldsymbol{\xi}_{\text {red }}$, mean values of the joint angles $\boldsymbol{q}$ (where $\dot{\boldsymbol{q}} \approx 0$ ) and mean values of associated base wrench components $\chi_{\text {sensor, } i}$ where $i=3,4,5$ were used for least-squares regression.

The stiffness identification procedure was also based on the reduced model where $\boldsymbol{q}_{d}$ and $\boldsymbol{\tau}_{d}$ was obtained by taking the mean values in the time interval before the onset of the disturbance. Analogously, a second interval for $\boldsymbol{q}$ and $\boldsymbol{\tau}$ was chosen after the displacement. For descriptive reasons, typical estimated joint stiffness matrices are transformed to Cartesian coordinates and visualised as stiffness ellipsoids in Figure 3. Each stiffness matrix $K_{q}$ was determined from 50 disturbance measurements; consequently each map was constructed from 50 sets of $8 \times 400$ data points. We preprocessed the EMG data with a full wave rectification and split the data into time windows of length 70 afterwards. For $\phi$ we picked the maximum along each of the signals followed by two layers of unsupervised feature extraction using the approach of [9]. We chose 100 soft rectified linear units as the nonlinearity in our model: $\sigma(x)=\ln (1+\exp x)$. All hyper parameters of the learning process were selected by
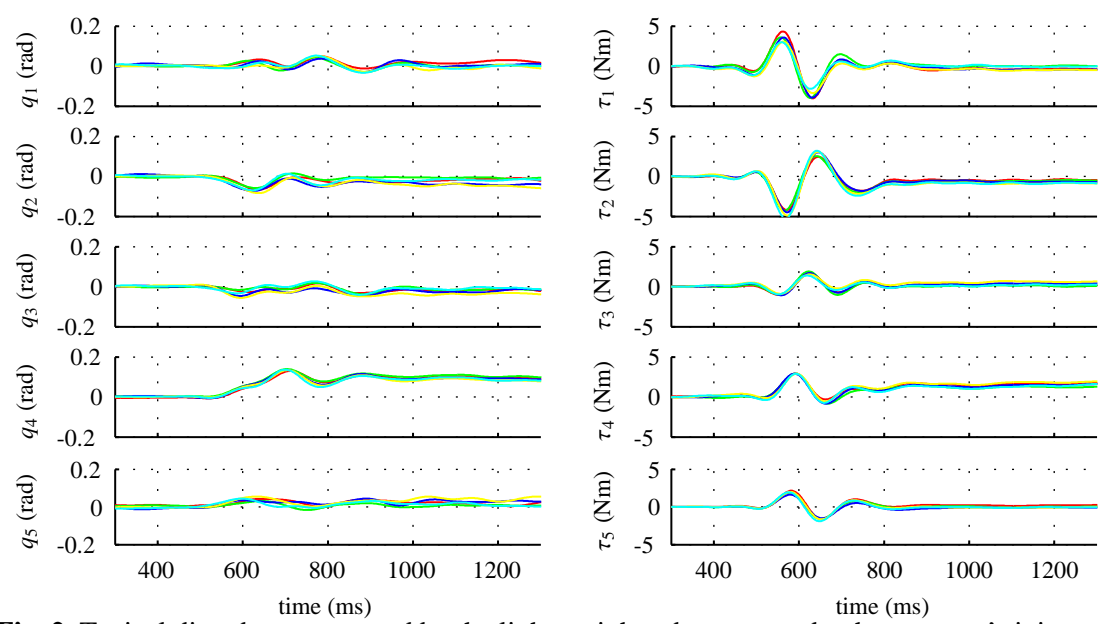

Fig. 2 Typical disturbances exerted by the light-weight robot, mapped to human arm's joint space. To demonstrate the repeatability the data is aligned along the time axis. 
random search and picking those which performed best on a held out validation set. The average normalized root mean squared error for the multi-layered model was 0.3378 . In contrast, a linear model never achieved 0.39 or better. For an example of the predicted stiffness matrices, see Figure 4.

\subsection{Comments}

Estimating joint stiffness in more than two degrees entails a chain of model assumptions, while model uncertainties accumulate from kinematics over inertial to stiffness identification. The identification of inertial parameters depend on measured joint angles $\boldsymbol{q}$ (and their derivatives) and are based on force / torque data measured on the mounting base, i.e., the measurement range must include human's whole body mass. These are affected by the following assumptions:

Fig. 3 Cartesian stiffness ellipsoids of estimated joint stiffness matrices. Each ellipsoid represents the force-field generated due to spherical displacements (here, the radius $r=5 \mathrm{~mm}$ ). The ellipsoid's origins are shifted to the point of pretension forces $\boldsymbol{F}=\left(F_{X}, F_{Y}, F_{Z}\right)^{T}$. Additionally, the principle axes (eigenvalues) of stiffness ellipsoids are displayed as straight lines.

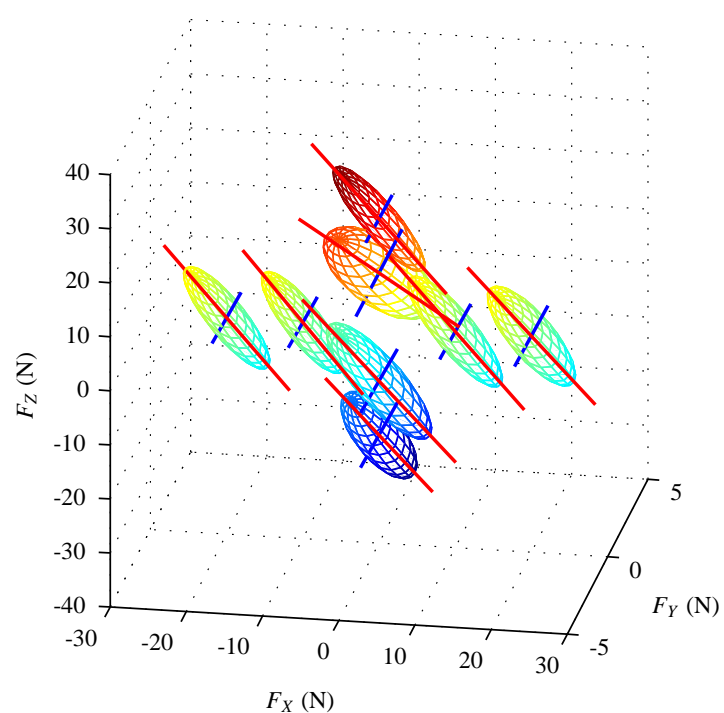

Fig. 4 Hinton diagrams of the stiffness matrices based on EMG prediction (left), estimation from force perturbations (middle) and the absolute value of their difference (right). Black boxes correspond to negative, white to positive values while the size represents the magnitude. Data was taken from the testing set. 
- For human arm's kinematics it is assumed that the shoulder joint is an ideal spherical joint and the elbow joint consist of two orthogonal, intersecting axes. Furthermore, it is assumed that optical markers placed on the upper and lower arm do not move relative to the skeleton. Both issues, extensively studied in [1], lead to biased estimates of joint angles $\boldsymbol{q}$ and their time derivatives.

- In particular for some small mass moments of inertia, the reaction force at the mounting base undercuts the sensitivity of the force / torque sensor.

- For the stiffness identification we assume that muscle activation $\boldsymbol{a}$ are constant. This implies fast perturbations, where displacements are stabilized in a short time (cf. [7]). Otherwise a change in activations would have changed the impedance.

This chain of assumptions forces us to analyze the results depicted in Fig. 3 intensively. For instance, from planar measurements it is known [5] that stiffness ellipses align their major axis in the direction of the pretension force applied. For the present estimations this effect can be observed only in the direction of $F_{Z}$.

\section{Main Experimental Insights}

In this work we have introduced a new and unique method to measure the stiffness of the human arm in 5-DoF joint space, viz. 3 shoulder DoF, the elbow flexion, and the lower arm rotation. Identification of arm kinematics and deriving the Jacobian matrix allows for transferring the measured joints stiffnesses to the Cartesian domain. We thus pioneered the measurement of human arm impedance in more than 2 Cartesian coordinates.

Furthermore, we have proposed and incorporated a multi-layered regression model which maps surface EMG signals to joint stiffness. With this method, combined with a detailed kinematic model, we can accurately estimate arm impedance without the need of mechanical perturbations. This is essential in order to determine human arm impedance not only in static positions but also along a trajectory during task execution, without the need of perturbation measurements.

Given this framework we are now able to investigate how humans modulates arm impedance in any task. The resulting measurements can be used to derive methods of impedance modulation for robotic arms.

\section{References}

1. Biryukova, E., Roby-Brami, A., Frolov, A., Mokhtari, M.: Kinematics of human arm reconstructed from spatial tracking system recordings. Journal of Biomechanics 33, 985-995 (2000)

2. Burdet, E., Osu, R., Franklin, D.W., Yoshioka, T., Milner, T.E., Kawato, M.: A method for measuring endpoint stiffness during multi-joint arm movements. Journal of Biomechanics 33, 1705-1709 (2000)

3. Chen, Y., McInroy, J.E.: Estimation of symmetric positive-definite matrices from imperfect measurements. IEEE Transaction on Automatic Control 47, 1721-1725 (2002) 
4. Gomi, H., Kawato, M.: Human arm stiffness and equilibrium-point trajectory during multijoint movement. Biological Cybernetics 76, 163-171 (1997)

5. Gomi, H., Osu, R.: Task-dependent viscoelasticity of human multijoint arm and its spatial characteristics for interaction with environments. The Journal of Neuroscience 18, 8965-8978 (1998)

6. Hogan, N.: The mechanics of multi-joint posture and movement control. Biological Cybernetics 52, 315-331 (1985)

7. Lakatos, D., Petit, F., van der Smagt, P.: Conditioning vs. excitation time for estimating impedance parameters of the human arm. In: IEEE Humanoids (2011)

8. Mussa-Ivaldi, F.A., Hogan, N., Bizzi, E.: Neural, mechanical, and geometric factors subserving arm posture in humans. The Journal of Neuroscience 5, 2732-2743 (1985)

9. Ngiam, J., Koh, P.W., Chen, Z., Bhaskar, S., Ng, A.: Sparse filtering. In: J. Shawe-Taylor, R. Zemel, P. Bartlett, F. Pereira, K. Weinberger (eds.) Advances in Neural Information Processing Systems 24, pp. 1125-1133 (2011)

10. Perreault, E.J., Kirsch, R.F., Acosta, A.M.: Multiple-input, multiple-output system identification for characterization of limb stiffness dynamics. Biological Cybernetics 80, 327-337 (1999)

11. Perreault, E.J., Kirsch, R.F., Crago, P.E.: Effects of voluntary force generation on the elastic components of endpoint stiffness. Experimental Brain Research 141, 312-323 (2001)

12. Tsuji, T., Morasso, P.G., Goto, K., Ito, K.: Human hand impedance characteristics during maintained posture. Biological Cybernetics 72, 475-485 (1995)

13. Venture, G., Yamane, K., Nakamura, Y., Yamamoto, T.: Identification of Human Limb Viscoelasticity using Robotics Methods to Support the Diagnosis of Neuromuscular Diseases. The International Journal of Robotics Research 28(10), 1322-1333 (2009) 